\title{
RESONANT ROBIN PROBLEMS WITH INDEFINITE AND UNBOUNDED POTENTIAL
}

\author{
Nikolaos S. Papageorgiou - George Smyrlis
}

\begin{abstract}
We study a semilinear Robin problem with an indefinite and unbounded potential and a reaction term which asymptotically at $\pm \infty$ is resonant with respect to any nonprincipal nonnegative eigenvalue. We prove two multiplicity theorems producing three and four nontrivial solutions respectively. Our approach uses variational methods based on the critical point theory, truncation and perturbation techniques, and Morse theory (critical groups).
\end{abstract}

\section{Introduction}

Let $\Omega \subseteq \mathbb{R}^{N}$ be a bounded domain with a $C^{2}$-boundary $\partial \Omega$. In this paper we study the following Robin problem:

(1.1) $-\Delta u(z)+\xi(z) u(z)=f(z, u(z)) \quad$ in $\Omega, \quad \frac{\partial u}{\partial n}+\beta(z) u=0 \quad$ on $\partial \Omega$.

Here $\xi \in L^{s}(\Omega)$ with $s>N$ and in general it is sign-changing (indefinite potential) and unbounded from below. The aim of this work is to prove multiplicity theorems for problem (2.1) when the reaction $f$ asymptotically as the second argument tends to $\pm \infty$ interacts with the spectrum of the differential operator $-\Delta u+\xi(z) u$ with Robin boundary conditions (resonant problems). In particular, we show that if $f(z, \cdot)$ at $\pm \infty$ is resonant with respect to any nonnegative and nonprincipal eigenvalue of the differential operator, then problem (2.1)

2010 Mathematics Subject Classification. 35J20, 35J60, 58E05.

Key words and phrases. Indefinite and unbounded potential; resonance; Robin boundary condition; maximum principle; critical groups, multiple solutions. 
admits at least three nontrivial solutions. Subsequently, by strengthening the conditions on $f(z, \cdot)$, we show the existence of at least four nontrivial solutions.

Multiplicity theorems for resonant Dirichlet problems with no potential term (that is, $\xi=0$ ), were proved by Bartsch and Wang [3], Castro, Cossio and Velez [4], Hofer [8], Liu and Li [10]. For resonant Neumann problems also with zero potential term, we mention the works of Gasinski and Papageorgiou [7], Motreanu, Motreanu and Papageorgiou [12] and Tang and Wu [19]. Recently there have been some multiplicity theorems for elliptic problems with an indefnite and unbounded potential. We mention the works of Kyritsi and Papageorgiou [9], Papageorgiou and Papalini [15] (Dirichelt problems) and Papageorgiou and Smyrlis [17] (Neumann problems). For the Robin problem, there is only the recent work of Papageorgiou and Radulescu [16], who examine a class of parametric coercive equations.

Our approach combines variational methods based on the critical point theory together with truncation and perturbation techniques and Morse theory (critical groups).

\section{Mathematical background}

Let $X$ be a Banach space and $X^{*}$ its topological dual. By $\langle\cdot, \cdot\rangle$ we denote the duality brackets for the pair $\left(X^{*}, X\right)$. Given $\varphi \in C^{1}(X, \mathbb{R})$, we say that $\varphi$ satisfies the "Cerami condition" (the "C-condition" for short), if the following is true:

"Every sequence $\left\{u_{n}\right\}_{n \geq 1} \subseteq X$ such that $\left\{\varphi\left(u_{n}\right)\right\}_{n \geq 1}$ is bounded and

$$
\left(1+\left\|u_{n}\right\|\right) \varphi^{\prime}\left(u_{n}\right) \rightarrow 0 \text { in } X^{*} \text { as } n \rightarrow \infty,
$$

admits a strongly convergent subsequence".

This is a compactness-type condition on the functional $\varphi$, it is more general than the usual "Palais-Smale condition" and it suffices to prove a deformation theorem and from it to derive the minimax theory of the critical values of $\varphi$. A basic result in this theory is the well-known "mountain pass theorem", which we state below in a slightly more general form (see, for example, Gasinski and Papageorgiou [6, p. 648]).

TheOREM 2.1. If $\varphi \in C^{1}(X)$ satisfies the $C$-condition, $u_{0}, u_{1} \in X, r>0$ are such that $\left\|u_{0}-u_{1}\right\|>r$ and

$$
\begin{gathered}
\max \left\{\varphi\left(u_{0}\right), \varphi\left(u_{1}\right)\right\}<\inf \left[\varphi(u):\left\|u-u_{0}\right\|=r\right]=m_{r}, \\
c=\inf _{\gamma \in \Gamma} \max _{0 \leq t \leq 1} \varphi(\gamma(t)), \quad \text { where } \Gamma=\left\{\gamma \in C([0,1], X): \gamma(0)=u_{0}, \gamma(1)=u_{1}\right\},
\end{gathered}
$$

then $c \geq m_{r}$ and $c$ is a critical value of $\varphi$.

For problem (2.1) the relevant function spaces are the Sobolev space $H^{1}(\Omega)$, the Banach space $C^{1}(\bar{\Omega})$ and the boundary Lebesgue spaces $L^{p}(\partial \Omega)(1 \leq p \leq \infty)$. 
In what follows, by $\|\cdot\|$ we denote the norm of the Sobolev space $H^{1}(\Omega)$ defined by

$$
\|u\|=\left[\|u\|_{2}^{2}+\|D u\|_{2}^{2}\right]^{1 / 2} \quad \text { for all } u \in H^{1}(\Omega) .
$$

The space $C^{1}(\bar{\Omega})$ is an ordered Banach space with positive cone

$$
C_{+}=\left\{u \in C^{1}(\bar{\Omega}): u(z) \geq 0 \text { for all } z \in \bar{\Omega}\right\} .
$$

This cone has a nonempty interior

$$
\operatorname{int} C_{+}=\left\{u \in C_{+}: u(z)>0 \text { for all } z \in \bar{\Omega}\right\} .
$$

On $\partial \Omega$ we introduce the $(N-1)$-dimensional Hausdorff measure (surface measure) denoted by $\sigma(\cdot)$. Using this measure, we can define the Lebesgue spaces $L^{p}(\partial \Omega)(1 \leq p \leq \infty)$. From the theory of Sobolev spaces, we know that there exists a unique continuous linear map $\gamma_{0}: H^{1}(\Omega) \rightarrow L^{2}(\partial \Omega)$, known as the "trace map", such that $\gamma_{0}(u)=\left.u\right|_{\partial \Omega}$ for all $u \in H^{1}(\Omega) \cap C(\bar{\Omega})$. The trace map $\gamma_{0}$ is compact into $L^{q}(\partial \Omega)$ for every $q \in[1,2(N-1) /(N-2))$. Moreover, we know that

$$
\operatorname{im} \gamma_{0}=H^{1 / 2,2}(\partial \Omega) \text { and } \operatorname{ker} \gamma_{0}=H_{0}^{1}(\Omega) .
$$

In what follows, for the sake of notational simplicity, we drop the use of the trace map $\gamma_{0}$. All Sobolev functions restricted on $\partial \Omega$ are understood in the sense of traces.

Consider a Caratheodory function $f_{0}: \Omega \times \mathbb{R} \rightarrow \mathbb{R}$ (that is, for all $x \in \mathbb{R}$, $z \mapsto f_{0}(z, x)$ is measurable and for almost all $z \in \Omega, x \mapsto f_{0}(z, x)$ is continuous) with subcritical growth in $x \in \mathbb{R}$,

$$
\left|f_{0}(z, x)\right| \leq a_{0}(z)\left(1+|x|^{r-1}\right) \quad \text { for a.a. } z \in \Omega \text {, all } x \in \mathbb{R},
$$

with $a_{0} \in L^{\infty}(\Omega)_{+}$and

$$
1<r<2^{*}= \begin{cases}2 N /(N-2) & \text { if } N \geq 3 \\ r \in(2, \infty) \text { arbitrarily big } & \text { if } N=2 \\ +\infty & \text { if } N=1\end{cases}
$$

We set

$$
F_{0}(z, x)=\int_{0}^{x} f_{0}(z, s) d s, \quad \vartheta(u)=\|D u\|_{2}^{2}+\int_{\Omega} \xi(z) u^{2} d z+\int_{\partial \Omega} \beta(z) u^{2} d \sigma
$$

for all $u \in H^{1}(\Omega)$ and consider the $C^{1}$-functional $\varphi_{0}: H^{1}(\Omega) \rightarrow \mathbb{R}$ defined by

$$
\varphi_{0}(u)=\frac{1}{2} \vartheta(u)-\int_{\Omega} F_{0}(z, u(z)) d z \quad \text { for all } u \in H^{1}(\Omega) .
$$

As in Papageorgiou and Radulescu [16], using the regularity result of Wang [20], we have 
Proposition 2.2. If $\xi \in L^{s}(\Omega)$ with $s>N, \beta \in W^{1, \infty}(\partial \Omega), \beta \geq 0, f_{0}$ is as above and $u_{0} \in H^{1}(\Omega)$ is a local $C^{1}(\bar{\Omega})$-minimizer of $\varphi_{0}$, that is, there exists $\rho_{0}>0$ such that

$$
\varphi_{0}\left(u_{0}\right) \leq \varphi_{0}\left(u_{0}+h\right) \quad \text { for all } h \in C^{1}(\bar{\Omega}) \text {, with }\|h\|_{C^{1}(\bar{\Omega})} \leq \rho_{0},
$$

then $u_{0} \in C^{1, \alpha}(\bar{\Omega})$ for some $\alpha \in(0,1)$ and $u_{0}$ is a local $H^{1}(\Omega)$-local minimizer of $\varphi_{0}$, that $i s$, there exists $\rho_{1}>0$ such that

$$
\varphi_{0}\left(u_{0}\right) \leq \varphi_{0}\left(u_{0}+h\right) \quad \text { for all } h \in H^{1}(\Omega) \text {, with }\|h\| \leq \rho_{1} .
$$

We will use spectral properties of the Robin differential operator of problem (2.1). For this reason, we consider the following linear eigenvalue problem:

$$
-\Delta u(z)+\xi(z) u(z)=\lambda u(z) \quad \text { in } \Omega, \quad \frac{\partial u}{\partial n}+\beta(z) u=0 \quad \text { on } \partial \Omega .
$$

Concerning the boundary weight $\beta$, the following condition will be in effect throughout the rest of this paper:

$$
\mathrm{H}(\beta) \beta \in W^{1, \infty}(\partial \Omega), \beta \geq 0 .
$$

Evidently, if $\beta \equiv 0$ then we have the Neumann problem. Therefore our analysis of (2.1) incorporates that in the work of Papageorgiou and Smyrlis [17]. In fact our arguments follow those of [17] with suitable modifications to incorporate the presence of the boundary term.

Proposition 2.3. If $\xi \in L^{N / 2}(\Omega)$ when $N \geq 3, \xi \in L^{s}(\Omega)$ with $s>1$ when $N=2, \xi \in L^{1}(\Omega)$ when $N=1$ and $H(\beta)$ holds, then

$$
\widehat{\lambda}_{1}=\inf \left[\vartheta(u): u \in H^{1}(\Omega),\|u\|_{2}=1\right]>-\infty .
$$

Proof. We argue indirectly. Suppose that $\widehat{\lambda}_{1}=-\infty$. Then we can find $\left\{u_{n}\right\}_{n \geq 1} \subseteq H^{1}(\Omega)$ such that

$$
\left\|u_{n}\right\|_{2}=1 \quad \text { for all } n \geq 1 \text { and } \vartheta\left(u_{n}\right) \rightarrow-\infty \quad \text { as } n \rightarrow \infty .
$$

So, we can find $n_{0} \in \mathbb{N}$ such that

$$
\vartheta\left(u_{n}\right) \leq-1 \quad \text { for all } n \geq n_{0} .
$$

Suppose that $\left\|u_{n}\right\| \rightarrow \infty$ as $n \rightarrow \infty$. We set $y_{n}=u_{n} /\left\|u_{n}\right\|$ for $n \geq 1$. Then $\left\|y_{n}\right\|=1$ for all $n \geq 1$ and so we may assume that

$$
y_{n} \stackrel{\mathrm{w}}{\longrightarrow} y \quad \text { in } H^{1}(\Omega) \text { and } y_{n} \rightarrow y \quad \text { in } L^{2}(\Omega) \text { and } L^{2}(\partial \Omega) \text {. }
$$

Note that, by the Sobolev embedding theorem, we have that $\left\{y_{n}^{2}\right\}_{n \geq 1} \subseteq L^{2^{*} / 2}(\Omega)$ is bounded. Therefore, we can say that $y_{n}^{2} \stackrel{\mathrm{w}}{\longrightarrow} y^{2}$ in $L^{2^{*} / 2}(\Omega)$ as $n \rightarrow \infty$ (see (2.4)). By hypothesis, $\xi \in L^{N / 2}(\Omega)$ and for $N \geq 3,2 / 2^{*}+2 / N=1$. So, we have

$$
\int_{\Omega} \xi(z) y_{n}^{2} d z \rightarrow \int_{\Omega} \xi(z) y^{2} d z \quad \text { as } n \rightarrow \infty
$$


Also, hypothesis $\mathrm{H}(\beta)$ and (2.4) imply that

$$
\int_{\partial \Omega} \beta(z) y_{n}^{2} d \sigma \rightarrow \int_{\partial \Omega} \beta(z) y^{2} d \sigma \quad \text { as } n \rightarrow \infty .
$$

Note that

$$
\begin{aligned}
\left\|y_{n}\right\|_{2}=\frac{\left\|u_{n}\right\|_{2}}{\left\|u_{n}\right\|}=\frac{1}{\left\|u_{n}\right\|} & \text { for all } n \geq 1 \quad(\text { see }(2.3)) \\
& \Rightarrow\|y\|_{2}=0 \quad(\text { see }(2.4)) \quad \text { and so } \quad y=0 .
\end{aligned}
$$

From (2.3) we have

$$
\vartheta\left(y_{n}\right) \leq-\frac{1}{\left\|u_{n}\right\|^{2}} \quad \text { for all } n \geq n_{0} \Rightarrow y_{n} \rightarrow 0 \quad \text { in } H^{1}(\Omega)
$$

(see (2.4)-(2.7)), which contradicts to the fact that $\left\|y_{n}\right\|=1$ for all $n \geq 1$. Hence $\left\{u_{n}\right\}_{n \geq 1} \subseteq H^{1}(\Omega)$ is bounded. By passing to a suitable subsequence if necessary, we have

$$
\begin{gathered}
u_{n} \stackrel{\mathrm{w}}{\longrightarrow} u \quad \text { in } H^{1}(\Omega), \quad \int_{\Omega} \xi(z) u_{n}^{2} d z \rightarrow \int_{\Omega} \xi(z) u^{2} d z, \\
\int_{\partial \Omega} \beta(z) u_{n}^{2} d \sigma \rightarrow \int_{\partial \Omega} \beta(z) u^{2} d \sigma .
\end{gathered}
$$

From (2.3) and (2.8), we have $\vartheta(u)=-\infty$, a contradiction. Therefore $\widehat{\lambda}_{1}>-\infty$.

REMARK 2.4. By the Lagrange multiplier rule, $\hat{\lambda}_{1}$ is the first eigenvalue of (2.1).

The next proposition will allow us to use the spectral theory of compact self-adjoint operators in order to analyze problem (2.1).

Proposition 2.5. If $\xi \in L^{N / 2}(\Omega)$ when $N \geq 3, \xi \in L^{s}(\Omega)$ with $s>1$ when $N=2, \xi \in L^{1}(\Omega)$ when $N=1$ and $\mathrm{H}(\beta)$ holds, then there exist $\mu>\max \left\{-\widehat{\lambda}_{1}, 0\right\}$ and $c_{0}>0$ such that

$$
\vartheta(u)+\mu\|u\|_{2}^{2} \geq c_{0}\|u\|^{2} \quad \text { for all } u \in H^{1}(\Omega) .
$$

Proof. Suppose that the statement does not hold. Then for every $n \in \mathbb{N}$ we can find $u_{n} \in H^{1}(\Omega)$ such that

$$
\vartheta\left(u_{n}\right)+n\left\|u_{n}\right\|_{2}^{2}<\frac{1}{n}\left\|u_{n}\right\|^{2} .
$$

Let $y_{n}=u_{n} /\left\|u_{n}\right\|, n \geq 1$. Then $\left\|y_{n}\right\|=1$ for all $n \geq 1$ and so, by passing to a suitable subsequence if necessary, we may assume that

$$
y_{n} \stackrel{\mathrm{w}}{\longrightarrow} y \quad \text { in } H^{1}(\Omega), \quad y_{n} \rightarrow y \quad \text { in } L^{2}(\Omega) \text { and } L^{2}(\partial \Omega) \text { as } n \rightarrow \infty .
$$

From (2.9) we have

$$
\vartheta\left(y_{n}\right)+n\left\|y_{n}\right\|_{2}^{2}<\frac{1}{n} \text { for all } n \geq 1 .
$$


Note that $\vartheta$ is sequentially weakly lower semicontinuous. So, from (2.10) we have

$$
\vartheta(y) \leq \liminf _{n \rightarrow \infty} \vartheta\left(y_{n}\right) .
$$

So, if in (2.11) we pass to the limit as $n \rightarrow \infty$ and use (2.12), then

$$
\begin{array}{ll}
\lim _{n \rightarrow \infty}\left(n\left\|y_{n}\right\|_{2}^{2}\right)=0, & \\
\Rightarrow y=0 & \\
\Rightarrow y_{n} \rightarrow 0 \quad \text { in } H^{1}(\Omega) & (\text { see }(2.10)),
\end{array}
$$

This contradicts to the fact that $\left\|y_{n}\right\|=1$ for all $n \geq 1$ and thus proves the proposition.

Having this proposition, we can introduce an equivalent inner product $(\cdot, \cdot)$ * on $H^{1}(\Omega)$, by setting for all $u, h \in H^{1}(\Omega)$,

$$
(u, y)_{*}=\int_{\Omega}(D u, D y)_{\mathbb{R}^{N}} d z+\int_{\Omega}(\xi(z)+\mu) u h d z+\int_{\partial \Omega} \beta(z) u h d \sigma .
$$

Given $g \in L^{2}(\Omega)$, by the Riesz representation theorem, we can find a unique $u \in H^{1}(\Omega)$ such that

$$
(u, h)_{*}=\int_{\Omega} g h d z \quad \text { for all } h \in H^{1}(\Omega) .
$$

This introduces a continuous linear map $K_{*}: L^{2}(\Omega) \rightarrow H^{1}(\Omega)$ defined by

$$
K_{*}(g)=u .
$$

Let $i: H^{1}(\Omega) \rightarrow L^{2}(\Omega)$ be the embedding map. By the Sobolev embedding theorem, we know that $i$ is compact. We have

$$
\left(\left(K_{*} \circ i\right)(v), h\right)_{*}=\int_{\Omega} v h d z \quad \text { for all } v, h \in H^{1}(\Omega) .
$$

Evidently, $K_{*} \circ i$ is compact and self-adjoint. Moreover, we have

$$
\left(\left(K_{*} \circ i\right)(v), v\right)_{*}=\|v\|_{2}^{2}>0 \quad \text { for all } v \in H^{1}(\Omega), v \neq 0 .
$$

Then, from the spectral theorem for compact self-adjoint positive operators (see, for example, Gasinski and Papageorgiou [6, p. 296]), we can find a sequence $\left\{\tau_{n}\right\}_{n \geq 1} \subseteq(0,+\infty)$ of eigenvalues of $K_{*} \circ i$ such that $\tau_{1}>\ldots>\tau_{n} \rightarrow 0^{+}$.

Let $\widehat{\lambda}_{n}=1 / \tau_{n}-\mu$ for all $n \geq 1$. Then $-\infty<\widehat{\lambda}_{1}<\ldots<\widehat{\lambda}_{n} \rightarrow+\infty$ as $n \rightarrow \infty$. Also, there is a corresponding sequence $\left\{\widehat{u}_{n}\right\}_{n \geq 1} \subseteq H^{1}(\Omega)$ of eigenfunctions (counting the multiplicity of each eigenvalue), which form an orthogonal basis of $H^{1}(\Omega)$ and an orthonormal basis of $L^{2}(\Omega)$. In fact, if $\xi \in L^{s}(\Omega)$ with $s>N$ then, using the regularity result of Wang [20], we can say that $\widehat{u}_{n} \in C^{1}(\bar{\Omega})$ for all $n \in \mathbb{N}$.

In what follows, by $E\left(\widehat{\lambda}_{n}\right)$ we denote the eigenspace corresponding to the eigenvalue $\widehat{\lambda}_{n}$. We know that $E\left(\widehat{\lambda}_{n}\right)$ is finite dimensional. Also, if $\xi \in L^{s}(\Omega)$ with $s>N$ then $E\left(\widehat{\lambda}_{n}\right) \subseteq C^{1}(\bar{\Omega})$. From de Figueiredo and Gossez [5], we know 
that $E\left(\widehat{\lambda}_{n}\right)$ has the so-called "Unique Continuation Property" (UCP for short), namely, if $u \in E\left(\widehat{\lambda}_{n}\right)$ and $u$ vanishes on a set of positive measure, then $u \equiv 0$.

We have the following orthogonal direct sum decomposition:

$$
H^{1}(\Omega)=\overline{\bigoplus_{n \geq 1} E\left(\widehat{\lambda}_{n}\right)}
$$

The eigenvalues $\left\{\widehat{\lambda}_{k}\right\}_{k \geq 1}$ have the following variational characterizations:

$$
\widehat{\lambda}_{1}=\inf \left[\frac{\vartheta(u)}{\|u\|_{2}^{2}}: u \in H^{1}(\Omega), u \neq 0\right]
$$

and for $m \geq 2$ we have

$$
\begin{aligned}
\widehat{\lambda}_{m} & =\inf \left[\frac{\vartheta(u)}{\|u\|_{2}^{2}}: u \in \overline{\bigoplus_{k \geq m} E\left(\widehat{\lambda}_{k}\right)}, u \neq 0\right] \\
& =\sup \left[\frac{\vartheta(u)}{\|u\|_{2}^{2}}: u \in \bigoplus_{k=1}^{m} E\left(\widehat{\lambda}_{k}\right), u \neq 0\right] .
\end{aligned}
$$

The infimum in $(2.15)$ is realized on $E\left(\widehat{\lambda}_{1}\right)$, while in $(2.16)$ both the infimum and the supremum are realized on $E\left(\widehat{\lambda}_{m}\right)$. From $(2.15)$ it is clear that the elements of $E\left(\widehat{\lambda}_{1}\right)$ do not change sign. In what follows, by $\widehat{u}_{1} \in H^{1}(\Omega)$ we denote the $L^{2}$-normalized (that is, $\left\|\widehat{u}_{1}\right\|_{2}=1$ ) positive eigenfunction corresponding to $\widehat{\lambda}_{1}$. If $\xi \in L^{s}(\Omega)$ with $s>N$ then $\widehat{u}_{1} \in C_{+} \backslash\{0\}$. Moreover, if $\xi^{+} \in L^{\infty}(\Omega)_{+}$then the maximum principle (see, for example, Gasinski and Papageorgiou [6, p. 738]) implies that $\widehat{u}_{1} \in \operatorname{int} C_{+}$. We mention that all the other eigenvalues have nodal (sign-changing) eigenfunctions (see, for example, Gasinski and Papageorgiou [6, p. 743]).

As an easy consequence of the variational characterizations (2.15) and (2.16) and of the UCP of the eigenspaces, we have the following useful inequalities.

Proposition 2.6. (a) If $\eta \in L^{\infty}(\Omega)$ and $\eta(z) \leq \widehat{\lambda}_{k}$ for almost all $z \in \Omega$ with strict inequality on a set of positive measure, then

$$
\vartheta(u)-\int_{\Omega} \eta(z) u^{2} d z \geq c_{1}\|u\|^{2} \quad \text { for some } c_{1}>0, \text { all } u \in \overline{\bigoplus_{n \geq k} E\left(\widehat{\lambda}_{n}\right)} .
$$

(b) If $\eta \in L^{\infty}(\Omega)$ and $\eta(z) \geq \widehat{\lambda}_{k}$ for almost all $z \in \Omega$ with strict inequality on a set of positive measure, then

$$
\vartheta(u)-\int_{\Omega} \eta(z) u^{2} d z \leq-c_{2}\|u\|^{2} \quad \text { for some } c_{2}>0, \text { all } u \in \bigoplus_{n=1}^{k} E\left(\widehat{\lambda}_{n}\right) .
$$

Let $m \in L^{\infty}(\Omega)_{+}, m \neq 0$. In addition to the eigenvalue problem (2.1), we consider also the following weighted version of it:

$(2.17)-\Delta u(z)+\xi(z) u(z)=\lambda m(z) u(z) \quad$ in $\Omega, \quad \frac{\partial u}{\partial n}+\beta(z) u=0 \quad$ on $\partial \Omega$. 
The same analysis and the same results are also valid for problem (2.17). The eigenvalues of (2.17) are denoted by $\widetilde{\lambda}_{k}(m), k \in \mathbb{N}$, to emphasize the dependence on the weight and in the variational characterizations (2.15) and (2.16) the quotient $\vartheta(u) /\|u\|_{2}^{2}$ is replaced by $\vartheta(u) / \int_{\Omega} m(z) u^{2} d z$.

From the general spectral theory (see, for example, Gasinski and Papageorgiou $[6$, p. 710$])$, we have that

$$
\tilde{\lambda}_{k}(m)=\inf _{Y \in \mathcal{L}_{k-1}} \sup _{u \in Y^{\perp}}\left[\vartheta(u): \int_{\Omega} m(z) u^{2} d z=1\right]
$$

with $\mathcal{L}_{k-1}$ being the family of all $(k-1)$-dimensional subspaces of $H^{1}(\Omega)$.

From (2.18) we are led to the following strict monotonicity property of the map $m \rightarrow \widetilde{\lambda}_{k}(m)$.

Proposition 2.7. If $m, m^{\prime} \in L^{\infty}(\Omega)_{+}, m(z) \leq m^{\prime}(z)$ for almost all $z \in \Omega$ and the inequality is strict on a set of positive measure, then $\widetilde{\lambda}_{k}\left(m^{\prime}\right)<\widetilde{\lambda}_{k}(m)$.

Next we recall some basic definitions and facts from Morse theory (critical groups) which we will need in the sequel. So, let $X$ be a Banach space, $\varphi \in$ $C^{1}(X, \mathbb{R})$ and $c \in \mathbb{R}$. We introduce the sets $\varphi^{c}=\{u \in X: \varphi(u) \leq c\}, K_{\varphi}=$ $\left\{u \in X: \varphi^{\prime}(u)=0\right\}$ and $K_{\varphi}^{c}=\left\{u \in K_{\varphi}: \varphi(u)=c\right\}$.

Let $\left(Y_{1}, Y_{2}\right)$ be a topological pair such that $Y_{2} \subseteq Y_{1} \subseteq X$. For every $k \in \mathbb{N}_{0}$, by $H_{k}\left(Y_{1}, Y_{2}\right)$ we denote the $k^{\text {th }}$-relative singular homology group for the topological pair $\left(Y_{1}, Y_{2}\right)$ with integer coefficients. Recall that for $k \in-\mathbb{N}$, $H_{k}\left(Y_{1}, Y_{2}\right)=0$. Let $u \in K_{\varphi}^{c}$ be isolated. The critical groups of $\varphi$ at $u \in X$ are defined by

$$
C_{k}(\varphi, u)=H_{k}\left(\varphi^{c} \cap U, \varphi^{c} \cap U \backslash\{u\}\right) \quad \text { for all } k \in \mathbb{N}_{0} .
$$

Here $U$ is a neighbuorhood of $u$ such that $K_{\varphi} \cap \varphi^{c} \cap U=\{u\}$. The excision property of singular homology theory implies that this definition of critical groups is independent of the choice of the neighbourhood $U$.

Suppose that $\varphi$ satisfies the C-condition and $\inf \varphi\left(K_{\varphi}\right)>-\infty$. Let $c<$ $\inf \varphi\left(K_{\varphi}\right)$. The critical groups of $\varphi$ at infinity are defined by

$$
C_{k}(\varphi, \infty)=H_{k}\left(X, \varphi^{c}\right) \quad \text { for all } k \in \mathbb{N}_{0} .
$$

The second deformation theorem (see, for example, Gasinski and Papageorgiou [6, p. 628]), implies that the above definition of critical groups at infinity, is independent of the choice of the level $c<\inf \varphi\left(K_{\varphi}\right)$.

Suppose that $K_{\varphi}$ is finite. We introduce the following quantities:

$$
\begin{aligned}
& M(t, u)=\sum_{k \geq 0} \operatorname{rank} C_{k}(\varphi, u) t^{k} \quad \text { for all } t \in \mathbb{R}, \text { all } u \in K_{\varphi}, \\
& P(t, \infty)=\sum_{k \geq 0} \operatorname{rank} C_{k}(\varphi, \infty) t^{k} \quad \text { for all } t \in \mathbb{R} .
\end{aligned}
$$


The "Morse relation" says that

$$
\sum_{u \in K_{\varphi}} M(t, u)=P(t, \infty)+(1+t) Q(t)
$$

where $Q(t)=\sum_{k \geq 0} \beta_{k} t^{k}$ is a formal series in $t \in \mathbb{R}$ with nonnegative integer coefficients $\beta_{k}$.

Recall that a Banach space $X$ has the "Kadec-Klee property", if the following is true:

$$
u_{n} \stackrel{\mathrm{w}}{\longrightarrow} u \quad \text { in } X \quad \text { and } \quad\left\|u_{n}\right\| \rightarrow\|u\| \Rightarrow u_{n} \rightarrow u \quad \text { in } X .
$$

If $x \in \mathbb{R}$, we set $x^{ \pm}=\max \{ \pm x, 0\}$. Then, for $u \in H^{1}(\Omega)$ we define $u^{ \pm}(\cdot)=$ $u(\cdot)^{ \pm}$. We have $u^{ \pm} \in H^{1}(\Omega), u=u^{+}-u^{-}$and $|u|=u^{+}+u^{-}$.

For every measurable function $h: \Omega \times \mathbb{R} \rightarrow \mathbb{R}$ (for example, a Carathéodory function), we set

$$
N_{h}(u)(\cdot)=h(\cdot, u(\cdot)) \text { for all } u \in H^{1}(\Omega)
$$

(the Nemytskiur map corresponding to $h$ ). By $|\cdot|_{N}$ we denote the Lebesgue measure on $\mathbb{R}^{N}$. Finally, let $m_{0}=\min \left[m \in \mathbb{N}: \widehat{\lambda}_{m} \geq 0\right]$.

\section{Three nontrivial solutions}

In this section we prove a multiplicity theorem producing three nontrivial smooth solutions for equations which at $\pm \infty$ can be resonant with respect to any nonprincipal, nonnegative eigenvalue $\widehat{\lambda}_{m}$.

We impose the following conditions on the potential $\xi$ and on the reaction term $f$ :

$\mathrm{H}(\xi) \xi \in L^{s}(\Omega)$ with $s>N$ if $N \geq 2$ and $s \geq 1$ if $N=1$ and $\xi^{+} \in L^{\infty}(\Omega)$.

$\left(\mathrm{H}_{1}\right) f: \Omega \times \mathbb{R} \rightarrow \mathbb{R}$ is a Carathéodory function such that $f(z, 0)=0$ for almost all $z \in \Omega$ and

(i) for every $\rho>0$, there exists $a_{\rho} \in L^{s}(\Omega), s>N$, such that

$$
|f(z, x)| \leq a_{\rho}(z) \quad \text { for a.a. } z \in \Omega \text {, all }|x| \leq \rho \text {; }
$$

(ii) there exist $m \geq \max \left\{m_{0}, 2\right\}$ and a function $\widehat{\eta} \in L^{\infty}(\Omega)$ such that

$\widehat{\eta}(z) \leq \widehat{\lambda}_{m+1} \quad$ for a.a. $z \in \Omega$, strictly on a set of positive measure,

$\widehat{\lambda}_{m} \leq \liminf _{x \rightarrow \pm \infty} \frac{f(z, x)}{x} \leq \limsup _{x \rightarrow \pm \infty} \frac{f(z, x)}{x} \leq \widehat{\eta}(z) \quad$ uniformly for a.a. $z \in \Omega ;$

(iii) if $F(z, x)=\int_{0}^{x} f(z, s) d s$, then $\lim _{x \rightarrow \pm \infty}[2 F(z, x)-x f(z, x)]=+\infty$ uniformly for almost all $z \in \Omega$;

(iv) there exists $\zeta_{0} \in L^{\infty}(\Omega)$ such that

$\zeta_{0}(z) \leq \widehat{\lambda}_{1} \quad$ for a.a. $z \in \Omega, \quad$ strictly on a set of positive measure, 


$$
\limsup _{x \rightarrow 0} \frac{f(z, x)}{x} \leq \zeta_{0}(z) \quad \text { uniformly for a.a. } z \in \Omega ;
$$

(v) for every $\rho>0$, there exists $\widehat{\xi}_{\rho}>0$ such that

$$
f(z, x) x+\widehat{\xi}_{\rho} x^{2} \geq 0 \quad \text { for a.a. } z \in \Omega \text {, all }|x| \leq \rho .
$$

Remarks 3.1. Hypothesis $\left(\mathrm{H}_{1}\right)$ (ii) says that asymptotically at $\pm \infty$, the quotient $f(z, x) / x$ reaches the spectral interval $\left[\widehat{\lambda}_{m}, \widehat{\lambda}_{m+1}\right]$. Resonance can occur with respect to $\widehat{\lambda}_{m}$ while with respect to the right endpoint $\widehat{\lambda}_{m+1}$ we have nonuniform nonresonance. Hypotheses $\left(\mathrm{H}_{1}\right)(\mathrm{iv}),(\mathrm{v})$ dictate a linear growth near zero.

ExAmPles 3.2. The following function satisfies hypotheses $\left(\mathrm{H}_{1}\right)$. For the sake of simplicity, we drop the $z$-dependence:

$$
f(x)= \begin{cases}\eta x-\gamma_{0} & \text { if } x<-1 \\ x\left(\gamma \cos \left(\pi x^{2} / 2\right)+c|x|\right) & \text { if }-1 \leq x \leq 1, \\ \eta x+\gamma_{0} & \text { if } x>1\end{cases}
$$

with $\gamma<\widehat{\lambda}_{1}, \eta \in\left[\widehat{\lambda}_{m}, \widehat{\lambda}_{m+1}\right)$ and $c=\eta+\gamma_{0}, \gamma_{0}>0$.

We introduce the following truncations-perturbations of the reaction $f(z, \cdot)$. Here $\mu>0$ is as in Proposition 2.5.

$$
\begin{aligned}
& \widehat{f}_{+}(z, x)= \begin{cases}0 & \text { if } x \leq 0, \\
f_{\mu}(z, x) & \text { if } 0<x,\end{cases} \\
& \widehat{f}_{-}(z, x)=f_{\mu}(z, x)-\widehat{f}_{+}(z, x),
\end{aligned}
$$

where $f_{\mu}(z, x)=f(z, x)+\mu x$. Both are Carathéodory functions. We set

$$
\widehat{F}_{ \pm}(z, x)=\int_{0}^{x} \widehat{f}_{ \pm}(z, s) d s
$$

and consider the $C^{1}$-functional $\widehat{\varphi}_{ \pm}: H^{1}(\Omega) \rightarrow \mathbb{R}$ defined by

$$
\widehat{\varphi}_{ \pm}(u)=\frac{1}{2} \vartheta(u)+\frac{\mu}{2}\|u\|_{2}^{2}-\int_{\Omega} \widehat{F}_{ \pm}(z, u) d z \quad \text { for all } u \in H^{1}(\Omega) .
$$

Also let $\varphi: H^{1}(\Omega) \rightarrow \mathbb{R}$ be the energy functional for problem (1.1) defined by

$$
\varphi(u)=\frac{1}{2} \vartheta(u)-\int_{\Omega} F(z, u) d z \quad \text { for all } u \in H^{1}(\Omega) .
$$

Evidently $\varphi \in C^{1}\left(H^{1}(\Omega)\right)$.

Proposition 3.3. If hypotheses $\mathrm{H}(\beta), \mathrm{H}(\xi)$ and $\left(\mathrm{H}_{1}\right)$ hold, then the functionals $\widehat{\varphi}_{ \pm}$satisfy the $C$-condition. 
Proof. We do the proof for $\widehat{\varphi}_{+}$, the proof for $\widehat{\varphi}_{-}$being similar. So, let $\left\{u_{n}\right\}_{n \geq 1} \subseteq H^{1}(\Omega)$ be a sequence such that

$$
\begin{aligned}
& \left|\widehat{\varphi}_{+}\left(u_{n}\right)\right| \leq M_{1} \quad \text { for some } M_{1}>0, \text { all } n \in \mathbb{N}, \\
& \left(1+\| u_{n}||\right) \widehat{\varphi}_{+}^{\prime}\left(u_{n}\right) \rightarrow 0 \quad \text { in } H^{1}(\Omega)^{*} \text { as } n \rightarrow \infty .
\end{aligned}
$$

From (3.3) we have

$$
\begin{aligned}
\mid\left\langle A\left(u_{n}\right), h\right\rangle+\int_{\Omega}(\xi(z)+\mu) u_{n} h d z+ & \int_{\partial \Omega} \beta(z) u_{n} h d \sigma \\
& -\int_{\Omega} \widehat{f}_{+}\left(z, u_{n}\right) h d z \mid \leq \frac{\varepsilon_{n}\|h\|}{1+\left\|u_{n}\right\|},
\end{aligned}
$$

for all $h \in H^{1}(\Omega)$, with $\varepsilon_{n} \downarrow 0$. Here $A \in \mathcal{L}\left(H^{1}(\Omega), H^{1}(\Omega)^{*}\right)$ is defined by

$$
\langle A(u), y\rangle=\int_{\Omega}(D u, D y)_{\mathbb{R}^{N}} d z \quad \text { for all } u, y \in H^{1}(\Omega) .
$$

In (3.4) we choose $h=-u_{n}^{-} \in H^{1}(\Omega)$ and obtain

$$
\begin{array}{cll}
\vartheta\left(u_{n}^{-}\right)+\mu\left\|u_{n}^{-}\right\|_{2}^{2} \leq \varepsilon_{n} & \text { for all } n \geq 1 & (\text { see }(3.1)), \\
\Rightarrow c_{0}\left\|u_{n}^{-}\right\|_{2}^{2} \leq \varepsilon_{n} & \text { for all } n \geq 1 & (\text { see Proposition 2.5) }, \\
\Rightarrow u_{n}^{-} \rightarrow 0 & \text { in } H^{1}(\Omega) \text { as } n \rightarrow \infty .
\end{array}
$$

Claim. $\left\{u_{n}^{+}\right\}_{n \geq 1} \subseteq H^{1}(\Omega)$ is bounded.

We argue by contradiction. Suppose that the claim is not true. Then by passing to a subsequence if necessary, we may assume that $\left\|u_{n}^{+}\right\| \rightarrow \infty$.

Let $y_{n}=u_{n}^{+} /\left\|u_{n}^{+}\right\|, n \geq 1$. Then $\left\|y_{n}\right\|=1, y_{n} \geq 0$ for all $n \geq 1$ and we may assume that

$$
y_{n} \stackrel{\mathrm{w}}{\longrightarrow} y \quad \text { in } H^{1}(\Omega) \quad \text { and } \quad y_{n} \rightarrow y \quad \text { in } L^{2 s /(s-1)}(\Omega) \text { and } L^{2}(\partial \Omega) \text {. }
$$

From (3.1), (3.4) and (3.5), we have

$$
\begin{array}{rl}
\mid\left\langle A\left(y_{n}\right), h\right\rangle+\int_{\Omega} \xi(z) y_{n} h & d z \\
& \quad+\int_{\partial \Omega} \beta(z) y_{n} h d z-\int_{\Omega} \frac{N_{f}\left(u_{n}^{+}\right)}{\left\|u_{n}^{+}\right\|} h d z \mid \leq \varepsilon_{n}^{\prime}\|h\|,
\end{array}
$$

for all $h \in H^{1}(\Omega)$, with $\varepsilon_{n}^{\prime} \rightarrow 0$. Hypotheses $\left(\mathrm{H}_{1}\right)$ (i), (ii) imply that

$$
\begin{aligned}
&|f(z, x)| \leq \widehat{a}(z)(1+|x|) \text { for a.a. } z \in \Omega, \text { all } x \in \mathbb{R}, \text { with } \widehat{a} \in L^{2}(\Omega)_{+}, \\
& \Rightarrow\left\{\frac{N_{f}\left(u_{n}^{+}\right)}{\left\|u_{n}^{+}\right\|}\right\}_{n \geq 1} \subseteq L^{2}(\Omega) \text { is bounded. }
\end{aligned}
$$


In (3.7) we choose $h=y_{n}-y \in H^{1}(\Omega)$, pass to the limit as $n \rightarrow \infty$ and use (3.6) and (3.9). Then

$$
\begin{gathered}
\lim _{n \rightarrow \infty}\left\langle A\left(y_{n}\right), y_{n}-y\right\rangle=0, \\
\Rightarrow\left\|D y_{n}\right\|_{2} \rightarrow\|D y\|_{2} \quad(\text { see }(3.6)) .
\end{gathered}
$$

Hilbert spaces have the Kadec-Klee property (it can be verified using the parallelogram law). So, from (3.6) and (3.10) we infer that

$$
\begin{gathered}
y_{n} \rightarrow y \quad \text { in } H^{1}(\Omega), \\
\Rightarrow\|y\|=1, \quad y \geq 0 .
\end{gathered}
$$

From (3.9) and hypothesis $\left(\mathrm{H}_{1}\right)$ (ii), at least for a subsequence, we have

$$
\frac{N_{f}\left(u_{n}^{+}\right)}{\left\|u_{n}^{+}\right\|} \stackrel{\mathrm{w}}{\longrightarrow} \eta y \quad \text { in } L^{2}(\Omega) \text { with } \widehat{\lambda}_{m} \leq \eta(z) \leq \widehat{\eta}(z) \text { for a.a. } z \in \Omega
$$

(see Aizicovici, Papageorgiou and Staicu [1], proof of Proposition 16). So, if in (3.7) we pass to the limit as $n \rightarrow \infty$ and use (3.6) and (3.13), then

$$
\langle A(y), h\rangle+\int_{\Omega} \xi(z) y h d z+\int_{\partial \Omega} \beta(z) y h d \sigma=\int_{\Omega} \eta(z) y h d z
$$

for all $h \in H^{1}(\Omega)$,

$$
\Rightarrow-\Delta y(z)+\xi(z) y(z)=\eta(z) y(z), \frac{\partial y}{\partial n}+\beta(z) y=0, \quad \text { on } \partial \Omega
$$

for almost all $z \in \Omega$ (see Papageorgiou and Radulescu [16]). From (3.13), (3.14) and Proposition 2.7, we have

$$
\widetilde{\lambda}_{m}(\eta) \leq \widetilde{\lambda}_{m}\left(\widehat{\lambda}_{m}\right)=1 \quad \text { and } \quad 1=\widetilde{\lambda}_{m+1}\left(\widehat{\lambda}_{m+1}\right)<\widetilde{\lambda}_{m+1}(\eta) .
$$

Returning to (3.14) and recalling that $m \geq 2$, we see that $y$ is either nodal or zero. Both contradict (3.12). This proves Claim.

Then (3.5) and Claim imply that $\left\{u_{n}\right\}_{n \geq 1} \subseteq H^{1}(\Omega)$ is bounded. So, as before, we may assume that

$$
u_{n} \stackrel{\mathrm{w}}{\longrightarrow} u \quad \text { in } H^{1}(\Omega) \quad \text { and } \quad u_{n} \rightarrow u \quad \text { in } L^{2 s /(s-1)}(\Omega) \text { and } L^{2}(\partial \Omega) .
$$

In (3.4) we choose $h=u_{n}-u \in H^{1}(\Omega)$, pass to the limit as $n \rightarrow \infty$ and use (3.15). Then

$$
\begin{array}{ll}
\lim _{n \rightarrow \infty}\left\langle A\left(u_{n}\right), u_{n}-u\right\rangle=0, & \\
\Rightarrow u_{n} \rightarrow u \text { in } H^{1}(\Omega) & \text { (as before via the Kadec-Klee property) }, \\
\Rightarrow \widehat{\varphi}_{+} & \text {satisfies the C-condition. }
\end{array}
$$

Similarly we show that $\widehat{\varphi}_{-}$satisfies the C-condition. 
Proposition 3.4. If hypotheses $\mathrm{H}(\beta), \mathrm{H}(\xi)$ and $\left(\mathrm{H}_{1}\right)$ hold, then the energy functional $\varphi$ satisfies the $C$-condition.

Proof. Let $\left\{u_{n}\right\}_{n \geq 1} \subseteq H^{1}(\Omega)$ be a sequence such that

$$
\begin{gathered}
\left|\varphi\left(u_{n}\right)\right| \leq M_{2} \quad \text { for some } M_{2}>0 \text { and all } n \geq 1, \\
\left(1+\| u_{n}||\right) \varphi^{\prime}\left(u_{n}\right) \rightarrow 0 \quad \text { in } H^{1}(\Omega)^{*} \text { as } n \rightarrow \infty .
\end{gathered}
$$

From (3.17) we have

$$
\begin{aligned}
\mid\left\langle A\left(u_{n}\right), h\right\rangle+\int_{\Omega} \xi(z) u_{n} h d z & +\int_{\partial \Omega} \beta(z) \\
& u_{n} h d \sigma \\
& -\int_{\Omega} f\left(z, u_{n}\right) h d z \mid \leq \frac{\varepsilon_{n}|| h \|}{1+\left\|u_{n}\right\|},
\end{aligned}
$$

for all $h \in H^{1}(\Omega)$, with $\varepsilon_{n} \rightarrow 0^{+}$. In (3.18) we choose $h=u_{n} \in H^{1}(\Omega)$. Then

$$
\vartheta\left(u_{n}\right)-\int_{\Omega} f\left(z, u_{n}\right) u_{n} d z \leq \varepsilon_{n} \quad \text { for all } n \geq 1 .
$$

On the other hand, from (3.16) we have

$$
-\vartheta\left(u_{n}\right)+\int_{\Omega} 2 F\left(z, u_{n}\right) d z \leq 2 M_{2} \text { for all } n \geq 1 .
$$

We add (3.19) and (3.20) and obtain

$$
\int_{\Omega}\left[2 F\left(z, u_{n}\right)-f\left(z, u_{n}\right) u_{n}\right] d z \leq M_{3} \text { for some } M_{3}>0 \text { and all } n \geq 1 .
$$

Claim. $\left\{u_{n}\right\}_{n \geq 1} \subseteq H^{1}(\Omega)$ is bounded.

We argue indirectly. Suppose that the claim is not true. Passing to a suitable subsequence if necessary, we may assume that $\left\|u_{n}\right\| \rightarrow \infty$. We introduce $y_{n}=$ $u_{n} /\left\|u_{n}\right\|, n \geq 1$. Then $\left\|y_{n}\right\|=1$ for all $n \geq 1$ and so we may assume that

$$
y_{n} \stackrel{\mathrm{w}}{\longrightarrow} y \quad \text { in } H^{1}(\Omega) \quad \text { and } \quad y_{n} \rightarrow y \quad \text { in } L^{2 s /(s-1)}(\Omega) \text { and } L^{2}(\partial \Omega) .
$$

From (3.18) we have

$$
\begin{aligned}
\mid\left\langle A\left(y_{n}\right), h\right\rangle+\int_{\Omega} \xi(z) y_{n} h d z+ & \int_{\partial \Omega} \beta(z) y_{n} h d \sigma \\
& -\int_{\Omega} \frac{N_{f}\left(u_{n}\right)}{\left\|u_{n}\right\|} h d z \mid \leq \frac{\varepsilon_{n}\|h\|}{\left\|u_{n}\right\|\left(1+\left\|u_{n}\right\|\right)},
\end{aligned}
$$

for all $n \geq 1$. Using (3.8), we see that

$$
\left\{\frac{N_{f}\left(u_{n}\right)}{\left\|u_{n}\right\|}\right\}_{n \geq 1} \subseteq L^{2}(\Omega) \text { is bounded. }
$$


From this and hypothesis $\left(\mathrm{H}_{1}\right)(\mathrm{ii})$, for at least a subsequence, we may assume that

$$
\frac{N_{f}\left(u_{n}\right)}{\left\|u_{n}\right\|} \stackrel{\mathrm{w}}{\longrightarrow} \eta y \quad \text { in } L^{2}(\Omega) \text { with } \widehat{\lambda}_{m} \leq \eta(z) \leq \widehat{\eta}(z) \text { for a.a. } z \in \Omega
$$

(see Aizicovici, Papageorgiou and Staicu [1]). In (3.23) we choose $h=y_{n}-y \in$ $H^{1}(\Omega)$, pass to the limit as $n \rightarrow \infty$ and use (3.22) and (3.24). Then

$$
\lim _{n \rightarrow \infty}\left\langle A\left(y_{n}\right), y_{n}-y\right\rangle=0
$$

$(3.25) \Rightarrow y_{n} \rightarrow y \quad$ in $H^{1}(\Omega)$ (via the Kadec-Klee property), hence $\|y\|=1$.

In (3.23) we pass to the limit as $n \rightarrow \infty$ and use (3.24), (3.25). Then

$$
\begin{gathered}
\langle A(y), h\rangle+\int_{\Omega} \xi(z) y h d z+\int_{\partial \Omega} \beta(z) y h d \sigma=\int_{\Omega} \eta(z) y h d z \quad \text { for all } h \in H^{1}(\Omega) \\
\quad \Rightarrow-\Delta y(z)+\xi(z) y(z)=\eta(z) y(z), \quad \frac{\partial y}{\partial n}+\beta(z) y=0, \quad \text { on } \partial \Omega
\end{gathered}
$$

for almost all $z \in \Omega$ (see Papageorgiou and Radulescu [16]).

From (3.24), we have $\widehat{\lambda}_{m} \leq \eta(z) \leq \widehat{\eta}(z) \leq \widehat{\lambda}_{m+1}$ for almost all $z \in \Omega$ and the last inequality is strict on a set of positive measure. First assume that the first inequality is also strict on a set of positive measure. Using Proposition 2.7, we have

$$
\widetilde{\lambda}_{m}(\eta)<\widetilde{\lambda}_{m}\left(\widehat{\lambda}_{m}\right)=1 \quad \text { and } \quad 1=\widetilde{\lambda}_{m+1}\left(\widehat{\lambda}_{m+1}\right)<\widetilde{\lambda}_{m+1}(\eta) .
$$

From (3.26) and (3.27) it follows that $y=0$, which contradicts (3.25).

Next, assume that $\widehat{\lambda}_{m}=\eta(z)$ for almost all $z \in \Omega$. From (3.25) and (3.26), we have

$$
\begin{aligned}
y & \in E\left(\widehat{\lambda}_{m}\right) \backslash\{0\}, \\
& \Rightarrow y(z) \neq 0 \quad \text { for a.a. } z \in \Omega \text { (by the UCP) } \\
& \Rightarrow\left|u_{n}(z)\right| \rightarrow \infty \quad \text { for a.a. } z \in \Omega, \\
& \Rightarrow 2 F\left(z, u_{n}(z)\right)-f\left(z, u_{n}(z)\right) u_{n}(z) \rightarrow+\infty \quad \text { for a.a. } z \in \Omega
\end{aligned}
$$

(see hypothesis $\left(\mathrm{H}_{1}\right)(\mathrm{iii})$ ).

From (3.28) and Fatou's lemma (note that hypotheses $\left(\mathrm{H}_{1}\right)(\mathrm{i})$, (iii) permit its use), we obtain

$$
\int_{\Omega}\left[2 F\left(z, u_{n}(z)\right)-f\left(z, u_{n}(z)\right) u_{n}(z)\right] d z \rightarrow+\infty \quad \text { as } n \rightarrow+\infty .
$$

Comparing (3.29) and (3.21), we have a contradiction. This proves Claim.

Due to Claim, we may assume that

$$
u_{n} \stackrel{\mathrm{w}}{\longrightarrow} u \quad \text { in } H^{1}(\Omega) \quad \text { and } \quad u_{n} \rightarrow u \quad \text { in } L^{2 s /(s-1)}(\Omega) \text { and } L^{2}(\partial \Omega) .
$$


We return to (3.18) and choose $h=u_{n}-u \in H^{1}(\Omega)$. Passing to the limit as $n \rightarrow \infty$ and using (3.30), we have

$$
\begin{aligned}
& \lim _{n \rightarrow \infty}\left\langle A\left(u_{n}\right), u_{n}-u\right\rangle=0 \\
& \Rightarrow u_{n} \rightarrow u \quad \text { in } H^{1}(\Omega) \quad \text { (by the Kadec-Klee property) } \\
& \Rightarrow \varphi \text { satisfies the C-condition. }
\end{aligned}
$$

Proposition 3.5. If hypotheses $\mathrm{H}(\beta), \mathrm{H}(\xi)$ and $\left(\mathrm{H}_{1}\right)$ hold, then $u=0$ is a local minimizer for the functionals $\widehat{\varphi}_{ \pm}$and $\varphi$.

Proof. We do the proof for the functional $\widehat{\varphi}_{+}$, the proofs for the functionals $\widehat{\varphi}_{-}$and $\varphi$ being similar. By virtue of hypothesis $\left(\mathrm{H}_{1}\right)(\mathrm{iv})$, given $\varepsilon>0$, we can find $\delta=\delta(\varepsilon)>0$ such that

$$
F(z, x) \leq \frac{1}{2}\left(\zeta_{0}(s)+\varepsilon\right) x^{2} \quad \text { for a.a. } z \in \Omega, \text { all }|x| \leq \delta_{0} .
$$

Let $u \in C^{1}(\bar{\Omega})$ with $\|u\|_{C^{1}(\bar{\Omega}} \leq \delta$. We have

$$
\begin{aligned}
\widehat{\varphi}_{+}(u) & =\frac{1}{2} \vartheta(u)+\frac{\mu}{2}\left\|u^{-}\right\|_{2}^{2}-\int_{\Omega} F\left(z, u^{+}\right) d z \\
& \geq \frac{1}{2}\left[\vartheta\left(u^{+}\right)-\int_{\Omega} \zeta_{0}(s)\left(u^{+}\right)^{2} d z-\varepsilon\left\|u^{+}\right\|^{2}\right]+\frac{1}{2} \vartheta\left(u^{-}\right)+\frac{\mu}{2}\left\|u^{-}\right\|_{2}^{2}
\end{aligned}
$$

$(\operatorname{see}(3.31))$

$$
\geq \frac{1}{2}\left(c_{1}-\varepsilon\right)\left\|u^{+}\right\|^{2}+\frac{1}{2} c_{0}\left\|u^{-}\right\|^{2}
$$

(see Propositions 2.5 and 2.6). Choosing $\varepsilon \in\left(0, c_{1}\right)$ from $(3.29)$ we infer that $u=0$ is a local $C^{1}(\bar{\Omega})$, local minimizer of $\widehat{\varphi}_{+}$. Using Proposition 2.2 , we conclude that $u=0$ is a local $H^{1}(\Omega)$, local minimizer of $\widehat{\varphi}_{+}$. Similarly for the functionals $\widehat{\varphi}-$ and $\varphi$.

Now we can produce two nontrivial constant sign solutions.

Proposition 3.6. If hypotheses $\mathrm{H}(\beta), \mathrm{H}(\xi)$ and $\left(\mathrm{H}_{1}\right)$ hold, then problem (1.1) has two nontrivial constant sign solutions

$$
u_{0} \in \operatorname{int} C_{+} \quad \text { and } \quad v_{0} \in-\operatorname{int} C_{+} .
$$

PROOF. It is straightforward to check that

$$
u \in K_{\widehat{\varphi}_{+}} \Rightarrow u \geq 0 \text { and } u \in K_{\widehat{\varphi}_{-}} \Rightarrow u \leq 0 .
$$

So, we assume that both sets are finite or otherwise we already have two sequences of distinct positive and negative solutions, which as we will see in the sequel belong in int $C_{+}$and in $-\operatorname{int} C_{+}$, respectively. 
First we produce the positive solution. From Proposition 3.5 and since $K_{\widehat{\varphi}_{+}}$ is finite, we can find $\rho \in(0,1)$ small such that

$$
0=\widehat{\varphi}_{+}(0)<\inf \left[\widehat{\varphi}_{+}(u):\|u\|=\rho\right]=\widehat{m}_{+}
$$

(see Aizicovici, Papageorgiou and Staicu [1], proof of Proposition 29).

Hypothesis $\left(\mathrm{H}_{1}\right)(\mathrm{ii})$, and since $m \geq 2$, implies that

$$
\left.\widehat{\varphi}_{+}\left(t \widehat{u}_{1}\right) \rightarrow-\infty \quad \text { as } t \rightarrow+\infty \quad \text { (recall that } \widehat{u}_{1} \in \operatorname{int} C_{+}\right) .
$$

Also, from Proposition 3.3 we know that the functional $\widehat{\varphi}_{+}$satisfies the Ccondition. This fact and (3.33), (3.34) permit the use of the mountain pass theorem (see Theorem 2.1). So, we can find $u_{0} \in H^{1}(\Omega)$ such that $u_{0} \in K_{\widehat{\varphi}_{+}}$ and $\widehat{m}_{+} \leq \widehat{\varphi}_{+}\left(u_{0}\right)$. Therefore $u_{0} \geq 0, u_{0} \neq 0$ (see (3.33)) and, if

$$
\left\langle A\left(u_{0}\right), h\right\rangle+\int_{\Omega} \xi(z) u_{0} h d z+\int_{\partial \Omega} \beta(z) u_{0} h d \sigma=\int_{\Omega} f\left(z, u_{0}\right) h d z
$$

for all $h \in H^{1}(\Omega)$, then

$$
\begin{aligned}
-\Delta u_{0}(z)+\xi(z) u_{0}(z) & =f\left(z, u_{0}(z)\right) & & \text { for a.a. } z \in \Omega, \\
\frac{\partial u_{0}}{\partial n}+\beta(z) u_{0} & =0 & & \text { on } \partial \Omega
\end{aligned}
$$

(see Papageorgiou and Radulescu [16]).

From hypotheses $\left(\mathrm{H}_{1}\right)(\mathrm{i})$, (ii), (iv), (v) we see that

$$
|f(z, x)| \leq \widehat{a}(z)|x| \quad \text { for a.a. } z \in \Omega, x \in \mathbb{R} \text {, with } \widehat{a} \in L^{s}(\Omega), s>N \text {. }
$$

We set

$$
\lambda(z)= \begin{cases}\frac{f\left(z, u_{0}(z)\right)}{u_{0}(z)} & \text { if } u_{0}(z) \neq 0 \\ 0 & \text { if } u_{0}(z)=0 .\end{cases}
$$

Evidently $\lambda \in L^{s}(\Omega)$ and we have

$$
-\Delta u_{0}(z)=(\lambda-\xi)(z) u_{0}(z) \quad \text { for a.a. } z \in \Omega, \quad \frac{\partial u_{0}}{\partial n}+\beta(z) u_{0}=0 \quad \text { on } \partial \Omega .
$$

Since $\lambda-\xi \in L^{s}(\Omega)$ (see hypothesis $\mathrm{H}(\xi)$ ), from Lemma 5.1 of Wang [20] we have $u_{0} \in L^{\infty}(\Omega)$ and so $\Delta u_{0} \in L^{s}(\Omega)$. Then Lemma 5.2 of Wang [20] (the CalderonZygmund estimates), implies that $u_{0} \in W^{2, s}(\Omega)$. Since $s>N$, from the Sobolev embedding theorem we have $W^{2, s}(\Omega) \hookrightarrow C^{1+\alpha}(\bar{\Omega})$ with $\alpha=1-N / s>0$. Hence $u_{0} \in C_{+} \backslash\{0\}$.

Let $\rho=\left\|u_{0}\right\|_{\infty}$ and let $\widehat{\xi}_{\rho}>0$ be as postulated by hypothesis $\left(\mathrm{H}_{1}\right)(\mathrm{v})$. Then

$$
f\left(z, u_{0}(z)\right)+\widehat{\xi}_{\rho} u_{0}(z) \geq 0 \text { for a.a. } z \in \Omega .
$$


So, we have

$$
\begin{array}{ll}
-\Delta u_{0}(z)+\left(\xi(z)+\widehat{\xi}_{\rho}\right) u_{0}(z) \geq 0 & \text { for a.a. } z \in \Omega, \\
\quad \Rightarrow \Delta u_{0}(z) \leq\left(\left\|\xi^{+}\right\|_{\infty}+\widehat{\xi}_{\rho}\right) u_{0}(z) & \text { for a.a. } z \in \Omega \quad \text { (see hypothesis } \mathrm{H}(\xi)) \\
\Rightarrow u_{0} \in \operatorname{int} C_{+} &
\end{array}
$$

(by the maximum principle, see Gasinski and Papageorgiou [6, p. 738]). Similarly, working with $\widehat{\varphi}_{-}$we produce a negative solution $v_{0} \in-\operatorname{int} C_{+}$.

To produce additional solutions, we need to strengthen the conditions on the reaction term $f(z, \cdot)$. More precisely, we assume the following conditions on the function $f$ :

$\left(\mathrm{H}_{2}\right) f: \Omega \times \mathbb{R} \rightarrow \mathbb{R}$ is a measurable function such that for almost all $z \in$ $\Omega, f(z, 0)=0, f(z, \cdot) \in C^{1}(\mathbb{R} \backslash\{0\})$ and

(i) $\left|f_{x}^{\prime}(z, x)\right| \leq a(z)\left(1+|x|^{r-1}\right)$ for almost all $z \in \Omega$, all $x \neq 0$, with $a \in L^{\infty}(\Omega)_{+}, c>0,1<r<2^{*}$;

(ii) there exist an integer $m \geq \max \left\{m_{0}, 2\right\}$ and a function $\widehat{\eta} \in L^{\infty}(\Omega)$ such that

- $\widehat{\eta}(z) \leq \widehat{\lambda}_{m+1}$ for almost all $z \in \Omega$, strictly on a set of positive measure,

- $\widehat{\lambda}_{m} \leq \liminf _{x \rightarrow \pm \infty} \frac{f(z, x)}{x} \leq \limsup _{x \rightarrow \pm \infty} \frac{f(z, x)}{x} \leq \widehat{\eta}(z)$ uniformly for almost all $z \in \Omega$

(iii) if $F(z, x)=\int_{0}^{x} f(z, s) d s$, then

$\lim _{x \rightarrow \pm \infty}[2 F(z, x)-f(z, x) x]=+\infty \quad$ uniformly for a.a. $z \in \Omega$;

(iv) $\left(f_{x}^{\prime}\right)_{+}(z, 0)=\lim _{x \rightarrow 0^{+}} \frac{f(z, x)}{x}$ and $\left(f_{x}^{\prime}\right)_{-}(z, 0)=\lim _{x \rightarrow 0^{-}} \frac{f(z, x)}{x}$ exist uniformly for almost all $z \in \Omega$, belong to $L^{\infty}(\Omega)$ and

$$
\left(f_{x}^{\prime}\right)_{+}(z, 0),\left(f_{x}^{\prime}\right)_{-}(z, 0) \leq \widehat{\lambda}_{1} \quad \text { for a.a. } z \in \Omega
$$

and the inequalities are strict on a set of positive measure.

REMARK 3.7. The stronger regularity condition on $f(z, \cdot)$ and hypothesis $\left(\mathrm{H}_{2}\right)$ (iv) imply that for every $\rho>0$, we can find $\widehat{\xi}_{\rho}>0$ such that $f(z, x) x+$ $\widehat{\xi}_{\rho} x^{2} \geq 0$ for almost all $z \in \Omega$, all $|x| \leq \rho$. So, in this case hypothesis $\left(\mathrm{H}_{1}\right)$ (iv) is automatically satisfied.

ExAmple 3.8. The example given after hypotheses $\left(\mathrm{H}_{1}\right)$, satisfies the new hypotheses $\left(\mathrm{H}_{2}\right)$ provided that in addition we assume that $\gamma_{0}=(\pi \gamma-\eta) / 2>0$. 
Another example is provided by the function $f$ below. Again for the sake of simplicity we drop the $z$-dependence:

$$
f(x)= \begin{cases}\widehat{\eta} x+\widehat{\lambda} & \text { if } x<-1 \\ \widehat{\gamma} x+\widehat{c}|x| \sin (\pi x / 2)-\widehat{\lambda} x^{2} & \text { if }-1 \leq x \leq 0, \\ \gamma x+c x \sin (\pi x / 2)-\lambda x^{2} & \text { if } 0 \leq x \leq 1 \\ \eta x+\lambda & \text { if } x>1\end{cases}
$$

with $\gamma, \widehat{\gamma}<\widehat{\lambda}_{1}, \eta, \widehat{\eta} \in\left[\widehat{\lambda}_{m}, \widehat{\lambda}_{m+1}\right), \lambda, \widehat{\lambda}>0$ and $c=\eta-\gamma+2 \lambda, \widehat{c}=\widehat{\eta}-\widehat{\gamma}-2 \widehat{\lambda}$.

We can prove the following three nontrivial solutions theorem.

THEOREM 3.9. If hypotheses $\mathrm{H}(\beta), \mathrm{H}(\xi)$ and $\left(\mathrm{H}_{2}\right)$ hold, then problem (1.1) admits at least three nontrivial solutions

$$
u_{0} \in \operatorname{int} C_{+}, \quad v_{0} \in-\operatorname{int} C_{+} \quad \text { and } \quad y_{0} \in C^{1}(\bar{\Omega}) .
$$

Proof. From Proposition 3.6, we already have two nontrivial solutions

$$
u_{0} \in \operatorname{int} C_{+}, \quad v_{0} \in-\operatorname{int} C_{+} .
$$

We consider the following orthogonal direct sum decomposition:

$$
H^{1}(\Omega)=\bar{H}_{m} \oplus \widehat{H}_{m+1},
$$

where

$$
\bar{H}_{m}=\bigoplus_{k=1}^{m} E\left(\widehat{\lambda}_{k}\right) \text { and } \quad \widehat{H}_{m+1}=\bar{H}_{m}^{\perp}=\bigoplus_{k \geq m+1} E\left(\widehat{\lambda}_{k}\right) .
$$

From hypotheses $\left(\mathrm{H}_{2}\right)(\mathrm{i})$, (ii), given $\varepsilon>0$, we can find $c_{3}=c_{3}(\varepsilon)>0$ such that

$$
F(z, x) \leq \frac{1}{2}(\widehat{\eta}(z)+\varepsilon) x^{2}+c_{3} \quad \text { for a.a. } z \in \Omega, \text { all } x \in \mathbb{R} .
$$

Then, for $u \in \widehat{H}_{m+1}$, we have

$$
\begin{aligned}
\varphi(u) & =\frac{1}{2} \vartheta(u)-\int_{\Omega} F(z, u) d z \\
& \geq \frac{1}{2}\left[\vartheta(u)-\int_{\Omega} \widehat{\eta}(z) u^{2} d z-\varepsilon\|u\|^{2}\right]-c_{3}|\Omega|_{N} \quad(\text { see }(3.35)) \\
& \geq \frac{1}{2}\left(c_{1}-\varepsilon\right)\|u\|^{2}-c_{3}|\Omega|_{N} \quad \text { (see Proposition 2.6). }
\end{aligned}
$$

Choosing $\varepsilon \in\left(0, c_{1}\right)$, from (3.36) we infer that if $\left.\varphi\right|_{\widehat{H}_{m+1}}$ is coercive then

$$
\inf _{\widehat{H}_{m+1}} \varphi>-\infty \text {. }
$$

Claim 1. $\widehat{\lambda}_{m} x^{2}-2 F(z, x) \rightarrow-\infty$ as $x \rightarrow \pm \infty$ uniformly for almost all $z \in \Omega$. 
From hypothesis $\left(\mathrm{H}_{2}\right)$ (iii) we see that, given any $\gamma>0$, we can find $M=$ $M(\gamma)>0$ such that

$$
f(z, x) x-2 F(z, x) \leq-\gamma \quad \text { for a.a. } z \in \Omega \text {, all } x \geq M .
$$

Then, from

$$
\frac{d}{d x} \frac{F(z, x)}{x^{2}}=\frac{f(z, x) x^{2}-2 x F(z, x)}{x^{4}}=\frac{f(z, x) x-2 F(z, x)}{x^{3}} \leq-\frac{\gamma}{x^{3}}
$$

for almost all $z \in \Omega$ and all $x \geq M$ (see (3.38)), we have

$$
\frac{F(z, x)}{x^{2}}-\frac{F(z, y)}{y^{2}} \leq \xi\left(\frac{1}{x^{2}}-\frac{1}{y^{2}}\right) \text { for a.a. } z \in \Omega \text {, all } x \geq y \geq M \text {. }
$$

Hypothesis $\left(\mathrm{H}_{2}\right)$ (ii) implies that

$$
\liminf _{x \rightarrow \pm \infty} \frac{F(z, x)}{x^{2}} \geq \frac{\widehat{\lambda}_{m}}{2} \quad \text { uniformly for a.a. } z \in \Omega .
$$

So, if in (3.39) we pass to the limit as $x \rightarrow+\infty$ and use (3.40), then

$$
\begin{aligned}
\frac{\widehat{\lambda}_{m}}{2}-\frac{F(z, y)}{y^{2}} \leq-\frac{\gamma}{y^{2}} \quad \text { for a.a. } z \in \Omega, \text { all } y \geq M, \\
\Rightarrow \widehat{\lambda}_{m} y^{2}-2 F(z, y) \leq-2 \gamma \quad \text { for a.a. } z \in \Omega, \text { all } y \geq M .
\end{aligned}
$$

Since $\gamma>0$ is arbitrary, from (3.41) we infer that

$$
\lim _{x \rightarrow+\infty}\left[\widehat{\lambda}_{m} x^{2}-2 F(z, x)\right]=-\infty \quad \text { uniformly for a.a. } z \in \Omega .
$$

In a similar fashion we show that

$$
\lim _{x \rightarrow-\infty}\left[\widehat{\lambda}_{m} x^{2}-2 F(z, x)\right]=-\infty \quad \text { uniformly for a.a. } z \in \Omega .
$$

This proves Claim 1.

Claim 2. $\left.\varphi\right|_{\bar{H}_{m}}$ is anticoercive (that is, if $\|u\| \rightarrow \infty$ for $u \in \bar{H}_{m}$, then $\varphi(u) \rightarrow-\infty)$.

Arguing by contradiction, suppose we could find $\left\{u_{n}\right\}_{n \geq 1} \subseteq \bar{H}_{m}$ and $\gamma>0$ such that

$$
-\gamma \leq \varphi\left(u_{n}\right) \text { for all } n \geq 1,\left\|u_{n}\right\| \rightarrow \infty \text {. }
$$

Let $y_{n}=u_{n} /\left\|u_{n}\right\|$. Then $y_{n} \in \bar{H}_{m}$ and $\left\|y_{n}\right\|=1$ for all $n \geq 1$. Since $\bar{H}_{m}$ is finite dimensional, we may assume that

$$
y_{n} \rightarrow y \quad \text { in } H^{1}(\Omega), \quad\|y\|=1 .
$$

From (3.42) we have

$$
-\frac{\gamma}{\left\|u_{n}\right\|^{2}} \leq \frac{1}{2} \vartheta\left(y_{n}\right)-\int_{\Omega} \frac{F\left(z, u_{n}\right)}{\left\|u_{n}\right\|^{2}} d z \quad \text { for all } n \geq 1 .
$$

From hypotheses $\left(\mathrm{H}_{2}\right)$ (i), (ii), (iv), it follows that

$$
|F(z, x)| \leq c_{4}\left(1+|x|^{2}\right) \quad \text { for a.a. } z \in \Omega \text {, all } x \in \mathbb{R} \text {, some } c_{4}>0 \text {. }
$$


Therefore we see that $\left\{F\left(\cdot, u_{n}(\cdot)\right) /\left\|u_{n}\right\|^{2}\right\}_{n \geq 1} \subseteq L^{1}(\Omega)$ is uniformly integrable. So, by the Dunford-Pettis theorem, passing to a subsequence if necessary and taking into account hypothesis $\left(\mathrm{H}_{2}\right)(\mathrm{ii})$, we have

$$
\frac{F\left(\cdot, u_{n}(\cdot)\right)}{\left\|u_{n}\right\|^{2}} \stackrel{\mathrm{w}}{\longrightarrow} \frac{1}{2} \eta y^{2} \quad \text { in } L^{1}(\Omega)
$$

with $\widehat{\lambda}_{m} \leq \eta(z) \leq \widehat{\eta}(z)$ for almost all $z \in \Omega$ (see Aizicovici, Papageorgiou and Staicu [1]). Hence, if in (3.44) we pass to the limit as $n \rightarrow \infty$ and use (3.43), (3.45), then

$$
0 \leq \frac{1}{2} \vartheta(y)-\frac{1}{2} \int_{\Omega} \eta(z) y^{2} d z .
$$

First suppose that the inequality $\widehat{\lambda}_{m} \leq \eta(z)$ for almost all $z \in \Omega$ (see (3.45)) is strict on a set of positive measure. Since $y \in \bar{H}_{m}$ (see (3.43)) and using Proposition 2.6, we have

$$
c_{2}\|y\|^{2} \leq 0 \Rightarrow y=0,
$$

which contradicts (3.43). So, we assume that $\eta(z)=\widehat{\lambda}_{m}$ for almost all $z \in \Omega$. From (2.16) and (3.43) we have

$$
\begin{aligned}
\vartheta(y)=\widehat{\lambda}_{m}\|y\|_{2}^{2} & \Rightarrow y \in E\left(\widehat{\lambda}_{m}\right) \backslash\{0\} & & \text { (recall that } \left.y \in \bar{H}_{m}\right) \\
& \Rightarrow y(z) \neq 0 & & \text { for a.a. } z \in \Omega(\text { by the UCP) } \\
& \Rightarrow\left|u_{n}(z)\right| \rightarrow+\infty & & \text { for a.a. } z \in \Omega .
\end{aligned}
$$

Using Claim 1 and Fatou's lemma, we have

$$
\int_{\Omega}\left[\widehat{\lambda}_{m} u^{2}-2 F\left(z, u_{n}\right)\right] d z \rightarrow-\infty \quad \text { as } n \rightarrow \infty .
$$

On the other hand, from (3.42) we have

$$
-2 \gamma \leq \vartheta\left(u_{n}\right)-\int_{\Omega} 2 F\left(z, u_{n}\right) d z \leq \widehat{\lambda}_{m}\left\|u_{n}\right\|^{2}-\int_{\Omega} 2 F\left(z, u_{n}\right) d z
$$

(recall that $u_{n} \in \bar{H}_{m}$ and see (2.16))

$$
=\int_{\Omega}\left[\widehat{\lambda}_{m} u_{n}^{2}-2 F\left(z, u_{n}\right)\right] d z \text { for all } n \geq 1 .
$$

Comparing (3.47) and (3.48), we have a contradiction. This proves Claim 2.

Due to (3.37) and Claim 2, we can use Proposition 6.63 of Motreanu, Motreanu and Papageorgiou [13, p. 160] and infer that

$$
C_{d_{m}}(\varphi, \infty) \neq 0 \quad \text { with } d_{m}=\operatorname{dim} \bar{H}_{m} \geq 2 .
$$

This implies that there exists $y_{0} \in K_{\varphi}$ such that

$$
C_{d_{m}}\left(\varphi, y_{0}\right) \neq 0 \quad \text { with } d_{m}=\operatorname{dim} \bar{H}_{m} \geq 2 \text {. }
$$


From Proposition 3.5 we have that $u=0$ is a local minimizer of $\varphi$. Hence

$$
C_{k}(\varphi, 0)=\delta_{k, 0} \mathbb{Z} \quad \text { for all } k \geq 0 .
$$

From (3.49) and (3.50) it follows that $y_{0} \neq 0$. From the proof of Proposition 3.6, we know that $u_{0} \in \operatorname{int} C_{+}$is a critical point of $\widehat{\varphi}_{+}$of mountain pass type. So, we have

$$
C_{1}\left(\widehat{\varphi}_{+}, u_{0}\right) \neq 0
$$

(see Motreanu, Motreanu and Papageorgiou [13, Proposition 6.100, p. 176]). From (3.1) it is clear that $\left.\widehat{\varphi}_{+}\right|_{C_{+}}=\left.\varphi\right|_{C_{+}}$. Since $u_{0} \in \operatorname{int} C_{+}$, we have

$$
\begin{aligned}
C_{k}\left(\left.\widehat{\varphi}_{+}\right|_{C^{1}(\bar{\Omega})}, u_{0}\right) & =C_{k}\left(\left.\varphi\right|_{C^{1}(\bar{\Omega})}, u_{0}\right) & & \text { for all } k \geq 0, \\
\Rightarrow C_{k}\left(\widehat{\varphi}_{+}, u_{0}\right) & =C_{k}\left(\varphi, u_{0}\right) & & \text { for all } k \geq 0 \text { (see Palais [14]) } \\
\Rightarrow \quad C_{1}\left(\varphi, u_{0}\right) & \neq 0 & & \text { (see }(3.51)) .
\end{aligned}
$$

Since $u_{0} \in \operatorname{int} C_{+}$and $\varphi \in C^{2-0}\left(H^{1}(\Omega)\right)$, from (3.52) and Theorem 2.7 of Li, Li and $\mathrm{Lu}[11]$, we have

$$
C_{k}\left(\varphi, u_{0}\right)=\delta_{k, 1} \mathbb{Z} \quad \text { for all } k \geq 0 .
$$

Similarly, using the functional $\widehat{\varphi}_{-}$we show that

$$
C_{k}\left(\varphi, v_{0}\right)=\delta_{k, 1} \mathbb{Z} \quad \text { for all } k \geq 0 .
$$

From (3.49), (3.50), (3.53), (3.54) it follows that

$$
y_{0} \notin\left\{0, u_{0}, v_{0}\right\} \Rightarrow y_{0} \text { is a third nontrivial solution of (1.1). }
$$

As before (see the proof of Proposition 3.6), using the regularity result of Wang [20], we have $y_{0} \in C^{1}(\bar{\Omega})$.

\section{Four nontrivial solutions}

In this section, we strengthen the conditions on the reaction term $f$ and prove a new multiplicity theorem producing four nontrivial solutions. The new hypotheses on the function $f$ are the following:

$\left(\mathrm{H}_{3}\right) f: \Omega \times \mathbb{R} \rightarrow \mathbb{R}$ is a measurable function such that for almost all $z \in$ $\Omega, f(z, 0)=0, f(z, \cdot) \in C^{1}(\mathbb{R} \backslash\{0\})$, hypotheses $\left(\mathrm{H}_{3}\right)$ (i), (iii), (iv) are the same as the corresponding hypotheses $\left(\mathrm{H}_{2}\right)(\mathrm{i})$, (iii), (iv) and

(ii) there exists an integer $m \geq \max \left\{m_{0}, 2\right\}$ such that

$$
\lim _{x \rightarrow \pm \infty} \frac{f(z, x)}{x}=\widehat{\lambda}_{m} \quad \text { uniformly for a.a. } z \in \Omega
$$

and

$$
\left(f(z, x)-\widehat{\lambda}_{m} x\right) x \geq 0, \quad \widehat{c}_{1}|x|^{q-1} \leq\left|f(z, x)-\widehat{\lambda}_{m} x\right| \leq \widehat{c}_{2}|x|^{q-1}
$$

for almost all $z \in \Omega$, all $|x| \geq M$, with $M, \widehat{c}_{1}, \widehat{c}_{2}>0$ and $q \in(1,2)$; 
(v) $f_{x}^{\prime}(z, x) \leq \widehat{\eta}(z)$ for almost all $z \in \Omega$, all $x \neq 0$ with $\widehat{\eta} \in L^{\infty}(\Omega)+$ as in $\left(\mathrm{H}_{3}\right)(\mathrm{ii})=\left(\mathrm{H}_{2}\right)(\mathrm{ii})$.

REMARK 4.1. The new extra hypothesis $\left(\mathrm{H}_{3}\right)(\mathrm{v})$ is satisfied if for example

$$
(f(z, x)-f(z, y))(x-y) \leq \widehat{\eta}(z)(x-y)^{2}
$$

for almost all $z \in \Omega$ and all $x, y \in \mathbb{R} \backslash\{0\}$.

The example given after hypotheses $\left(\mathrm{H}_{2}\right)$ satisfies $\left(\mathrm{H}_{3}\right)$ provided $2 \gamma+3 c \leq \eta$.

THeOREM 4.2. If hypotheses $\mathrm{H}(\beta), \mathrm{H}(\xi)$ and $\left(\mathrm{H}_{3}\right)$ hold, then problem (1.1) admits at least four nontrivial solutions

$$
u_{0} \in \operatorname{int} C_{+}, \quad v_{0} \in-\operatorname{int} C_{+} \quad \text { and } \quad y_{0}, \widehat{y} \in C^{1}(\bar{\Omega}) .
$$

Proof. From Theorem 3.9 we already have three nontrivial solutions

$$
u_{0} \in \operatorname{int} C_{+}, \quad v_{0} \in-\operatorname{int} C_{+} \quad \text { and } \quad y_{0} \in C^{1}(\bar{\Omega}) .
$$

From the proof of Theorem 3.9, we know that

$$
C_{d_{m}}\left(\varphi, y_{0}\right) \neq 0 \quad \text { with } d_{m}=\operatorname{dim} \bar{H}_{m} \geq 2 .
$$

For every $h \in \widehat{H}_{m+1}=\overline{\bigoplus_{k \geq m+1} E\left(\widehat{\lambda}_{k}\right)}$, we have

$$
\begin{array}{rlrl}
\left\langle\varphi^{\prime \prime}\left(y_{0}\right) h, h\right\rangle & =\vartheta(h)-\int_{\Omega} f_{x}^{\prime}\left(z, y_{0}\right) h^{2} d z & \\
& \geq \vartheta(y)-\int_{\Omega} \widehat{\eta} h^{2} d z & & \left(\text { see hypothesis }\left(\mathrm{H}_{3}\right)(\mathrm{v})\right) \\
& \geq c_{1}\|h\|^{2} & & (\text { see Proposition 2.6) }
\end{array}
$$

Then from the shifting theorem for $C^{2-0}$-functions (see Li, Li and Liu [11, Theorem 2.2]), we have

$$
C_{k}\left(\varphi, y_{0}\right)=\delta_{k, d_{m}} \mathbb{Z} \quad \text { for all } k \geq 0 .
$$

Let $f(z, x)=\widehat{\lambda}_{m} x+f_{0}(z, x)$ with $f_{0}(z, x)$ a Carathéodory function such that

$$
\lim _{x \rightarrow \pm \infty} \frac{f_{0}(z, x)}{x}=0 \quad \text { uniformly for a.a. } z \in \Omega
$$

(see hypothesis $\left.\left(\mathrm{H}_{3}\right)(\mathrm{ii})\right)$. Let $\widehat{A} \in \mathcal{L}\left(H^{1}(\Omega), H^{1}(\Omega)^{*}\right)$ be defined by

$$
\langle\widehat{A}(u), h\rangle=\int_{\Omega}(D u, D h)_{\mathbb{R}^{N}} d z+\int_{\Omega}\left(\xi(z)-\widehat{\lambda}_{m}\right) u h d z+\int_{\partial \Omega} \beta(z) u h d \sigma
$$

for all $u, h \in H^{1}(\Omega)$. This is a self-adjoint operator with 0 isolated in its spectrum. Moreover, we have $\left.\widehat{A}\right|_{\bar{H}_{m-1}}<0$ and $\left.\widehat{A}\right|_{\bar{H}_{m+1}}>0$. So $d_{m-1}=\operatorname{dim} \bar{H}_{m-1}$ is the Morse index of $\widehat{A}$ at infinity and $d_{m-1}+\operatorname{dim} E\left(\widehat{\lambda}_{m}\right)=d_{m}=\operatorname{dim} \bar{H}_{m}$ is the extended Morse index of $\widehat{A}$ at infinity (that is, $\operatorname{dim} E\left(\widehat{\lambda}_{m}\right)$ is the nullity of $\widehat{A}$ at infinity). 
Let $F_{0}(z, x)=\int_{0}^{x} f_{0}(z, u) d u$ and let $\psi: H^{1}(\Omega) \rightarrow \mathbb{R}$ be the $C^{1}$-functional defined by

$$
\psi(u)=\int_{\Omega} F_{0}(z, u(z)) d z \quad \text { for all } u \in H^{1}(\Omega) .
$$

Evidently $\psi(u) /\|u\|^{2} \rightarrow 0$ as $\|u\| \rightarrow \infty$ and we have

$$
\varphi(u)=\frac{1}{2}\langle A(u), u\rangle+\psi(u) \quad \text { for all } u \in H^{1}(\Omega) .
$$

Since $\psi$ is bounded and as usual we assume that $K_{\varphi}$ is finite (otherwise we already have an infinity of solutions), we see that condition $\left(A_{\infty}\right)$ in Motreanu, Motreanu and Papageorgiou [13, p. 164] and Bartsch and Li [2] is satisfied (see also the Remark on p. 178 of [13]). Moreover, hypothesis $\left(\mathrm{H}_{3}\right)$ (ii) and Lemma 2.1 of $\mathrm{Su}$ and Tang [18] imply that the angle condition $\left(A_{\infty}^{-}\right)$in Motreanu, Motreanu and Papageorgiou [13, p. 165] is satisfied. So Theorem 6.73 (b) of [13] implies that

$$
C_{k}(\varphi, \infty)=\delta_{k, d_{m}} \mathbb{Z} \quad \text { for all } k \in \mathbb{N}_{0} .
$$

From the proof of Theorem 3.9 we know that

$$
\begin{aligned}
C_{k}(\varphi, 0) & =\delta_{k, 0} \mathbb{Z} & & \text { for all } k \in \mathbb{N}_{0}, \\
C_{k}\left(\varphi, u_{0}\right) & =C_{k}\left(\varphi, v_{0}\right)=\delta_{k, 1} \mathbb{Z} & & \text { for all } k \in \mathbb{N}_{0} .
\end{aligned}
$$

Suppose that $K_{\varphi}=\left\{0, u_{0}, v_{0}, y_{0}\right\}$. Then from (4.2)-(4.5) and the Morse relation with $t=-1$, we have

$$
(-1)^{0}+2(-1)^{1}+(-1)^{d_{m}}=(-1)^{d_{m}} \Rightarrow(-1)=0,
$$

a contradiction. So, there exists $\widehat{y} \in K_{\varphi}, \widehat{y} \notin\left\{0, u_{0}, v_{0}, y_{0}\right\}$ and $\widehat{y} \in C^{1}(\bar{\Omega})$ (see Wang [20]). This is the fourth nontrivial solution.

Acknowledgements. The authors express their gratitude to the referee for his/her thoughtful corrections and remarks that improved the paper considerably.

\section{REFERENCES}

[1] S. Aizicovici, N.S. Papageorgiou and V. Staicu, Degree Theory for Operators of Monotone Type and Nonlinear Elliptic Equations with Inequality Constraints, Mem. Amer. Math. Soc., Vol. 196, No. 915, November 2008.

[2] T. BARTSCH AND S. LI, Critical point theory for asymptotically quadratic functionals and applications to problems with resonance, Nonlinear Anal. 28 (1997), 419-441.

[3] T. BARTSCH AND Z.Q. WANG, On the existence of sign changing solutions for semilinear Dirichlet problems, Topol. Methods Nonlinear Anal. 7 (1996), 115-131.

[4] A. Castro, J. Cossio and C. Velez, Existence of seven solutions for asymptotically linear Dirichlet problems without symmetries, Ann. Mat. Pura Appl. 192 (2013), 607619.

[5] D. de Figueiredo And J.P. Gossez, Strict monotonicity of eigenvalues and unique continuation, Comm. Partial Differential Equations 17 (1992), 339-346. 
[6] L. Gasinski and N.S. Papageorgiou, Nonlinear Analysis, Chapman Hall, CRC, Boca Raton, Fl (2006).

[7] _ Pairs of nontrivial solutions for Neumann problems, J. Math. Anal. Appl. 398 (2013), 649-663.

[8] H. Hofer, Variational and topological methods in partially ordered Hilbert spaces, Math. Ann. 261 (1982), 493-514.

[9] S. Kyritsi and N.S. Papageorgiou, Multiple solutions for Dirichlet problems with an indefinite potential, Ann. Mat. Pura Appl. 192 (2013), 297-315.

[10] S. LiU AND S. LI, Critical groups at infinity, saddle point reduction and elliptic resonant problems, Commun. Contemp. Math. 5 (2003), 761-773.

[11] C. Li, S. Li And J. Liu, Splitting theorem, Poincaré-Hopf theorem and jumping nonlinear problems, J. Funct. Anal. 221 (2005), 439-455.

[12] D. Motreanu, V.V. Motreanu and N.S. Papageorgiou, On resonant Neumann problems, Math. Ann. 354 (2012), 1117-1145.

[13] _ Topological and Variatonal Methods with Applications to Nonlinear Boundary Value Problems, Springer, New York (2014).

[14] R. Palais, Homotopy theory for infinite dimensional manifolds, Topology 5 (1966), 1-16.

[15] N.S. PAPageorgiou AND F. PAPALini, Seven solutions with sign information for sublinear equations with unbounded and indefinite potential and no symmetries, Israel J. Math. 201 (2014), 761-796.

[16] N.S. Papageorgiou and V. Radulescu, Multiple solutions with precise sign for nonlinear parametric Robin problems, J. Differential Equations 256 (2014), 2449-2479.

[17] N.S. Papageorgiou And G. Smyrlis, On a class of parametric Neumann problems with indefinite and unbounded potential, Forum Math. DOI: 10.1515/forum-2012-0042.

[18] J. Su AND C. TANG, Multiplicity results for semilinear elliptic equations with resonance at higher eigenvalues, Nonlinear Anal. 44 (2001), 311-321.

[19] C.-L. TANG AND X.P. Wu, Existence and multiplicity for solutions of Neumann problems for elliptic equations, J. Math. Anal. Appl. 288 (2003), 660-670.

[20] X. WANG, Neumann problems of semilinear elliptic equations involving critical Sobolev exponents, J. Differential Equations 93 (1991), 283-310.

Manuscript received July 1, 2015 accepted December 6, 2015

Nikolaos S. Papageorgiou and George Smyrlis

National Technical University of Athens

Department of Mathematics

Zografou Campus

Athens 157 80, GREECE

E-mail address: npapg@math.ntua.gr, gsmyrlis@math.ntua.gr 